\title{
Engineered Campylobacter jejuni Cas9 variant with enhanced activity
}

\section{Ryoya Nakagawa}

University of Tokyo

\section{Soh Ishiguro}

University of Tokyo

\section{Sae Okazaki}

University of Tokyo

\section{Hideto Mori}

University of Tokyo

Mamoru Tanaka

University of Tokyo

Hiroyuki Aburatani

University of Tokyo https://orcid.org/0000-0003-0438-1544

Nozomu Yachie

University of Tokyo

Hiroshi Nishimasu

University of Tokyo

Osamu Nureki ( $\nabla$ nureki@bs.s.u-tokyo.ac.jp )

University of Tokyo https://orcid.org/0000-0003-1813-7008

\section{Article}

Keywords: Gene-editing Tools, Adeno-associated Virus Vector, Therapeutic Genome Editing, Biochemical Characterization, Cleavage Activity

Posted Date: May 19th, 2021

DOl: https://doi.org/10.21203/rs.3.rs-512474/v1

License: (c) (i) This work is licensed under a Creative Commons Attribution 4.0 International License. Read Full License

Version of Record: A version of this preprint was published at Communications Biology on March 8th, 2022. See the published version at https://doi.org/10.1038/s42003-022-03149-7. 


\section{Abstract}

The RNA-guided DNA endonuclease Cas9 is a versatile genome-editing tool. However, the molecular weight of the commonly used Streptococcus pyogenes Cas9 is relatively large. Consequently, its gene cannot be efficiently packaged into an adeno-associated virus vector, thereby limiting its applications for therapeutic genome editing. Here, we biochemically characterized the compact Cas9 from Campylobacter jejuni (CjCas9) and found that CjCas9 has a previously unrecognized preference for the N3VRYAC protospacer adjacent motif. We thus rationally engineered a CjCas9 variant (enCjCas9), which exhibits enhanced cleavage activity and a broader targeting range both in vitro and in human cells, as compared with CjCas9. Furthermore, a nickase version of enCjCas9, but not CjCas9, fused with a cytosine deaminase mediated C-to-T conversions in human cells. Overall, our findings expand the CRISPR-Cas toolbox for therapeutic genome engineering.

\section{Introduction}

The RNA-guided DNA endonuclease Cas9 binds a single-guide RNA (sgRNA) and cleaves double-stranded DNA targets complementary to the RNA guide ${ }^{1}$ (Supplementary Fig. 1a), thus participating in the microbial CRISPR-Cas adaptive immune system ${ }^{2}$. Streptococcus pyogenes Cas9 (SpCas9) is the most widely used endonuclease for genome editing in eukaryotic cells ${ }^{3}$, and requires an NGG $(N=A / T / G / C)$ protospacer adjacent motif (PAM) downstream of the target sequence for DNA recognition and unwinding ${ }^{1,4}$. Since the catalytically inactive SpCas9 (dSpCas9) serves as an RNA-guided DNA-binding protein, dSpCas9 fusions with various proteins have been applied to new technologies, such as transcriptional regulation, epigenome editing, and chromosomal imaging ${ }^{5}$. In addition, base editors, comprising the SpCas9 D10A nickase (nSpCas9) fused to a cytosine or adenosine deaminase, have enabled C-to-T or A-to-G substitutions at target genomic sites in a guide RNA-dependent manner ${ }^{6}$.

Campylobacter jejuni Cas9 (CjCas9) is a 984 residue protein and is significantly smaller than SpCas9 $(1,368 \text { residues })^{7}$. Since CjCas 9 with an sgRNA can be packaged into an adeno-associated virus vector more efficiently, as compared with SpCas9, the compact CjCas9 is useful for in vivo therapeutic genome editing ${ }^{8-11}$. Previous studies revealed functional and structural differences between CjCas 9 and SpCas 9 8,12. While SpCas9 uses sgRNAs with 20-nucleotide (nt) guide lengths, CjCas9 requires 22-nt guide lengths for robust DNA cleavage in human cells ${ }^{8}$. In addition, CjCas9 recognizes the NNNNRYAC $(R=$ $\mathrm{A} / \mathrm{G} ; \mathrm{Y}=\mathrm{T} / \mathrm{C}) \mathrm{PAM}^{8}$ and the NNNVRYM $(\mathrm{V}=\mathrm{A} / \mathrm{T} / \mathrm{G} ; \mathrm{M}=\mathrm{A} / \mathrm{C}) \mathrm{PAM}^{12}$. While SpCas9 recognizes the NGG PAM in the non-target DNA strand through Arg1333 and Arg1335 ${ }^{13}$ (Supplementary Fig. 1b), CjCas9 uses a distinct set of amino-acid residues to form base-specific contacts with the PAM nucleotides in both the target and non-target strands ${ }^{12}$ (Supplementary Fig. 1c). However, some questions about the enzymatic properties of CjCas9 have remained unanswered. First, the optimal guide lengths for CjCas 9 were determined in human cells ${ }^{8}$, but not in vitro. Second, the potential preference of CjCas 9 for promiscuous PAM sequences has remained uninvestigated. These uncertainties might have hampered the wide use of CjCas 9 as a versatile genome-editing tool. In addition, unlike SpCas ${ }^{14-16}$ and Streptococcus aureus 
Cas9 17,18 , CjCas9 has not been generally harnessed for base editing, although CjCas 9 robustly induced indels in mammalian cells ${ }^{8}$.

In this study, we performed the functional characterization and rational engineering of CjCas9. Our biochemical analysis revealed that CjCas9 exhibits strong activity with an optimal 22-nt guide sgRNA and recognizes $\mathrm{N}_{3}$ VRYAC PAMs with unexpected nucleotide preferences. We rationally engineered a CjCas 9 variant (enCjCas9), which exhibits enhanced cleavage activity and an expanded targeting scope. The enCjCas 9 induced indels at endogenous target sites in human cells more efficiently, as compared with the wild-type CjCas9. Notably, a nickase version of enCjCas9, but not wild-type CjCas9, fused to a cytosine deaminase mediated C-to-T conversions at target sites in human cells. Collectively, our findings substantially enhance the utility of the compact CjCas9 in genome- and base-editing technologies.

\section{Results}

\section{Biochemical characterization of CjCas9}

To examine the optimal guide length for CjCas9, we performed in vitro cleavage experiments, using the purified CjCas9, sgRNAs with 20- to 23-nt guide segments, and linearized plasmid DNA containing a target sequence and the canonical $T_{3} A A C A C$ PAM. CjCas9 with the 20-nt guide sgRNA did not cleave the target DNA (Supplementary Fig. 2a,b). In contrast, CjCas9 with the 21-23-nt guide sgRNAs efficiently cleaved the target DNA, and the 22-nt guide sgRNA was optimal (Supplementary Fig. 2a,b), consistent with a previous study showing that 22-nt guide sgRNAs are ideal for CjCas9-mediated genome editing in human cells ${ }^{8}$.

Since we previously examined the DNA cleavage activities of CjCas9 with a 20-nt guide sgRNA ${ }^{12}$, we reanalyzed them using the optimal 22-nt guide sgRNA toward target DNAs with 16 different PAMs, in which the fourth to eighth nucleotides in the canonical $T_{3}$ AACAC PAM were individually substituted. CjCas 9 efficiently cleaved the target DNAs with the $T_{3}$ VACAC PAMs, but not that with the $T_{3}$ TACAC PAM (Fig. 1a, Supplementary Fig. 3a-c), confirming the importance of the fourth $V$ in the PAM. CjCas9 cleaved the target DNAs with the $T_{3} A R C A C$ and $T_{3} A A Y A C$ PAMs, but not those with the $T_{3} A Y C A C$ and $T_{3} A A R A C$ PAMs (Fig. 1a, Supplementary Fig. 3a-c), confirming the requirements of the fifth $\mathrm{R}$ and sixth $\mathrm{Y}$ for the PAM recognition. CjCas9 cleaved the target DNA with the $T_{3} A A C A C$ PAM more efficiently than with the $T_{3} A A C B D(B=T / G / C ; D=A / T / G)$ PAMs, indicating the importance of the seventh $A$ and eighth $C$. The crystal structure of the CjCas9-guide RNA-target DNA complex suggested that the seventh A:T and eighth C:G (modeled) pairs in the PAM duplex are recognized by Arg866 through hydrogen bonds with the PAM-complementary T and G nucleobases ${ }^{12}$ (Supplementary Fig. 3d), explaining the preference for the seventh $A$ and the eighth $C$ in the PAM. Together, these results revealed that CjCas9 recognizes the $\mathrm{N}_{3}$ VRYAC PAMs, and are essentially consistent with previous studies 8,12 . 
To examine the potential preference of CjCas9 for specific sequences in the $\mathrm{N}_{3}$ VRYAC PAMs, we measured the DNA cleavage activities of CjCas 9 toward 12 different DNA targets encompassing all possible 12 nucleotide combinations at the fourth to sixth positions in the $\mathrm{N}_{3}$ VRYAC PAMs. CjCas 9 showed reduced activities toward the $T_{3}$ VGYAC targets (in particular, the $T_{3} C G Y A C$ targets) relative to the $\mathrm{T}_{3}$ VAYAC targets, although they are included in the $\mathrm{N}_{3}$ VRYAC consensus sequences (Fig. $1 \mathrm{~b}$,

Supplementary Fig. $3 \mathrm{e}-\mathrm{g}$ ). Collectively, these results indicated that CjCas9 disfavors some combinations in the $\mathrm{N}_{3}$ VRYAC PAMs, such as $\mathrm{N}_{3}$ RGCAC and $\mathrm{N}_{3}$ CGYAC.

\section{Engineering of the enhanced CjCas9 variant}

To eliminate the bias of CjCas9-mediated PAM recognition, we sought to engineer a CjCas9 variant with enhanced activity. Previous studies revealed that additional interactions between Cas9 and the nucleic acids improve the DNA cleavage activity ${ }^{19,20}$. We thus introduced mutations that could form new interactions with the guide RNA or the target DNA, based on the crystal structure of CjCas $9{ }^{12}$ (Supplementary Fig. 5a). We purified more than 40 CjCas9 variants, and measured their cleavage activities toward the sub-optimal $\mathrm{T}_{3}$ VGCAC targets (Supplementary Fig. 5b). Notably, the L58Y and D900K mutations enhanced the DNA cleavage activity, and the L58Y/D900K double mutation further improved the activity of CjCas9 (Supplementary Fig. 5c-f). The crystal structure suggested that Tyr58 (L58Y) and Lys900 (D900K) interact with the guide RNA and the target DNA, respectively (Supplementary Fig. 5a). We will hereafter refer to the L58Y/D900K variant as the enhanced CjCas9 (enCjCas9).

We next compared the in vitro cleavage activities of the wild-type CjCas9 (referred to as CjCas9 for simplicity) and enCjCas9 toward 23 DNA targets with different PAMs. Unlike CjCas9, enCjCas9 efficiently cleaved all of the $T_{3}$ VRYAC targets, including the sub-optimal $T_{3}$ CGYAC targets (Fig. 1c,d, Supplementary Fig. $5 a-f)$. Furthermore, enCjCas 9 cleaved some non- $\mathrm{N}_{3}$ VRYAC targets, such as $\mathrm{T}_{3}$ TACAC and $\mathrm{T}_{3} A A C A D$ (Fig. 1c, Supplementary Fig. 5 a-c).

To comprehensively compare the PAM specificities of CjCas9 and enCjCas9, we performed in vitro PAM discovery assays, in which a DNA library containing the target sequence adjacent to a randomized 8-bp sequence was cleaved by the purified CjCas9 (CjCas9 or enCjCas9) with the 22-nt guide sgRNA, followed by deep sequencing of the cleavage products. The sequence logos of the 8-bp random sequences depleted in this assay revealed similar $\mathrm{N}_{3}$ VRYAC PAM sequences for CjCas9 and enCjCas9, although enCjCas9 exhibited more relaxed nucleotide requirements for the fifth, seventh, and eighth PAM positions (Fig. 1e,f). However, a sequence logo representation lacks detailed information about the preferences for individual sequences within promiscuous PAMs, although it is widely used to identify functional PAMs. We thus expressed the obtained sequencing data as $2 \mathrm{D}$ profiles of the mean $\log _{2}$ PAM depletion values on all 1,024 sequences at the fourth to eighth PAM positions. The PAM profiles revealed that CjCas9 has preferences among the $\mathrm{N}_{3}$ VRYAC PAMs (Fig. 1g), consistent with our in vitro cleavage data for the 
individual PAM targets. In contrast, enCjCas9 efficiently recognized the $\mathrm{N}_{3} \mathrm{VRYAC}$ PAMs and some non$\mathrm{N}_{3}$ VRYAC sequences, such as $\mathrm{N}_{3}$ VAYTC and $\mathrm{N}_{3}$ VAYGC (Fig. 1h).

\section{Genome editing in human cells}

To assess the activities of CjCas 9 and enCjCas9 in mammalian cells, we measured indel formation induced by CjCas9 or enCjCas 9 at 38 endogenous target sites with the optimal $\mathrm{N}_{3} \mathrm{VACAC}$, sup-optimal $\mathrm{N}_{3}$ VGCAC, and non- $\mathrm{N}_{3}$ VRYAC ( $\mathrm{N}_{3}$ TACAC and $\mathrm{N}_{3}$ AACAD) PAMs in human embryonic kidney (HEK) 293Ta cells. CjCas9 induced indels at the target sites with the optimal $\mathrm{N}_{3}$ VACA, sub-optimal $\mathrm{N}_{3}$ VGCAC, and non$\mathrm{N}_{3}$ VRYAC PAMs at $44.0-53.6 \%$ (48.5\% on average), $10.1-20.7 \%$ (16.4\% on average), and $3.1-20.8 \%$ (12.9\% on average) frequencies, respectively (Fig. 2a,b). In contrast, enCjCas9 induced indels at the optimal $\mathrm{N}_{3}$ VACAC, sub-optimal $\mathrm{N}_{3}$ VGCAC, and non- $\mathrm{N}_{3}$ VRYAC PAM sites at $58.4-68.7 \%(64.8 \%$ on average), $20.2-39.8 \%$ (32.3\% on average), and $14.8-41.0 \%$ (29.0\% on average) frequencies, respectively (Fig. 2a,b). These results demonstrated that, as compared with CjCas9, enCjCas9 exhibits higher cleavage activities and broader targeting ranges in human cells, consistent with our in vitro cleavage data. Next, we compared the genome-editing efficiencies of CjCas9 and enCjCas9 with those of SpCas9 at two target sites with NGGAACAC PAMs, which can be accessed by both CjCas9 $\left(\mathrm{N}_{3}\right.$ VRYAC PAM) and SpCas9 (NGG PAM). CjCas9, enCjCas9, and SpCas9 generated indels at these two sites with $70.3-86.6 \%(78.4 \%$ on average), $79.7-87.5 \%$ (83.6\% on average), and $55.8-62.6 \%$ (59.2\% on average) frequencies, respectively (Fig. 2c). These results indicated that CjCas9 with optimal 22-nt guide sgRNAs can induce indels at target sites with appropriate PAMs, at efficiencies comparable to or higher than those of SpCas9, as previously reported ${ }^{8}$.

\section{Base editing in human cells}

Target-AID, comprising the SpCas9 D10A nickase mutant fused to the Petromyzon marinus cytosine deaminase 1 (PmCDA1) and the uracil DNA glycosylase inhibitor (UGI), mediates C-to-T conversion at target genomic sites ${ }^{15}$. We replaced the SpCas9 D10A nickase in Target-AID (referred to as SpCas9-AID for comparison) with the CjCas9 D8A and enCjCas9 D8A nickases to create CjCas9-AID and enCjCas9AID, respectively. We examined whether CjCas9-AID and enCjCas9-AID could mediate C-to-T conversions at 38 endogenous target sites (identical to those tested for indel formation) in HEK293 cells.

Unexpectedly, CjCas9-AID did not induce C-to-T conversions at the tested target sites (Fig. 3a,b). In contrast, enCjCas9-AID induced C-to-T conversions at 20 target sites at $>5 \%$ frequencies (the optimal $\mathrm{N}_{3}$ VACAC, sub-optimal $\mathrm{N}_{3}$ VGCAC, and non- $\mathrm{N}_{3}$ VRYAC sites at $15.8-25.1 \%$ (21.5\% on average), 4.3-13.2\% (8.0\% on average), and $1.2-12.4 \%$ (6.2\% on average) frequencies, respectively) (Fig. 3a,b). We also compared the base-editing efficiencies of CjCas9-AID, enCjCas9-AID, and SpCas9-AID at the two target sites with the NGGAACAC PAMs. Unlike CjCas9-AID, enCjCas9-AID induced C-to-T conversions at these target sites, albeit at lower efficiencies than those of SpCas9-AID (Fig. 3c). enCjCas9-AID induced C-to-T conversions predominantly at the -21 to -8 positions in target sites (Supplementary Fig. 6). These data revealed that enCjCas9, but not CjCas9, can be harnessed for base editing technologies. 


\section{Discussion}

In this report, we biochemically characterized the compact CjCas9, and found that CjCas9 efficiently cleaves a target DNA with a 22-nt guide sgRNA, consistent with a previous study in human cells ${ }^{8}$. In contrast, CjCas9 failed to cleave a target DNA with a 20-nt guide sgRNA, while SpCas9 exhibited robust DNA cleavage activity with 20-nt guide sgRNAs. These results reinforce the notion that the optimal guide lengths are different among Cas9 enzymes, as previously reported ${ }^{8,21,22}$. A structural comparison of the Cas9-guide RNA-target DNA complexes suggested that these functional differences are related to structural differences in their REC domains, which interact with the $5 \mathrm{C}$ end of their guide RNAs ${ }^{22}$, although the precise mechanisms remain unknown. We also found that CjCas9 has a previously unrecognized bias for $\mathrm{N}_{3}$ VRYAC PAMs, and has reduced activities toward target sites with sub-optimal PAMs, such as $\mathrm{N}_{3}$ VGYAC. Although CjCas 9 exhibited similar trends in PAM preferences in vitro and in human cells, the genome and base-editing activities of CjCas9 were highly dependent on the target loci, probably due to their different genomic contexts, such as chromatin states. These results highlight the importance of in vitro cleavage experiments for the accurate determination of the PAM specificity, as described previously ${ }^{23-25}$. In addition, our results demonstrate that a 2D PAM profile ${ }^{24,25}$ or a PAM wheel 26 , rather than a 1D sequence logo, is critical to fully visualize functional PAM sequences, particularly when Cas9 recognizes relatively long, promiscuous PAMs. Collectively, our findings underscore the practical importance of the use of optimal 22-nt guide sgRNAs and the selection of appropriate PAMs in CjCas9-mediated genome engineering applications.

Based on the structural information, we introduced the L58Y and D900K mutations into CjCas9 to create the enCjCas 9 variant with enhanced activity. The crystal structure of CjCas9 ${ }^{12}$ suggests that Lys 900 (D900K) interacts with the backbone phosphate of the target DNA, as observed in the engineered Cas9 variants, such as FnCas9-RHA ${ }^{19}$ and SpCas9-NG ${ }^{20}$. In contrast, Tyr58 (L58Y) apparently forms a stacking interaction with $\mathrm{U} 48$ in the guide RNA, which is similar to that between the equivalent Tyr72 and U50 in the SpCas9 structure ${ }^{27}$, indicating that additional interactions with the guide RNA can also contribute to facilitating Cas9-mediated DNA cleavage. These results suggest that the reinforcement of protein-nucleic-acid interactions is a widely applicable and effective strategy to engineer CRISPR-Cas enzymes with enhanced activity.

We found that CjCas9 edits target genomic sites with optimal $\mathrm{N}_{3}$ VRYAC PAMs as efficiently as SpCas9, as previously reported ${ }^{8}$. In contrast, CjCas9-AID failed to induce C-to-T conversions even at the identical target sites, consistent with the fact that CjCas9-based cytosine base editors are not currently available, while a CjCas9-based adenosine base editor was reported recently ${ }^{28}$. Notably, we determined that enCjCas9-AID, unlike CjCas9-AID, can induce C-to-T conversions at target sites in human cells. Given that the compact enCjCas9-AID (1,437 residues, $4.3 \mathrm{~kb})$ can be packaged into an adeno-associated virus vector, enCjCas9-AID would be useful for in vivo base editing, thus expanding the utility of the CRISPRCas toolbox. It will be important to examine whether the enCjCas9 nickase can be combined with other cytosine deaminases ${ }^{14,17,18}$ and adenosine deaminases ${ }^{16}$. Furthermore, the catalytically inactive 
enCjCas9 could serve as a compact RNA-guided DNA-binding platform applicable to other technologies, such as transcriptional regulation.

\section{Methods}

\section{Sample preparation}

The CjCas9 proteins were prepared, as described previously. Briefly, Escherichia coli Rosetta 2 (DE3) cells (Novagen) were transformed with the modified pE-SUMO-CjCas 9 vector ${ }^{12}$, and then cultured at $37^{\circ} \mathrm{C}$ in LB medium (containing $20 \mathrm{mg} / \mathrm{L}$ kanamycin) until the $\mathrm{OD}_{600}$ reached 0.8 . After the addition of $0.1 \mathrm{mM}$ isopropyl $\beta$-D-thiogalactopyranoside (Nacalai Tesque), the E. coli cells were incubated at $20^{\circ} \mathrm{C}$ for $20 \mathrm{~h}$, and then harvested by centrifugation. The $E$. coli cells were resuspended in buffer $\mathrm{A}(20 \mathrm{mM}$ Tris- $\mathrm{HCl}, \mathrm{pH}$ 8.0, $20 \mathrm{mM}$ imidazole, and $1 \mathrm{M} \mathrm{NaCl}$ ), lysed by sonication, and then centrifuged. The supernatant was mixed with Ni-NTA Superflow resin (QIAGEN), and the mixture was loaded into a Poly-Prep Column (BioRad). The protein was eluted with buffer $\mathrm{B}(20 \mathrm{mM}$ Tris- $\mathrm{HCl}, \mathrm{pH} 8.0,0.3 \mathrm{M}$ imidazole, and 0.3 M NaCl). To remove the $\mathrm{His}_{6}$-SUMO-tag, the purified protein was mixed with SUMO protease and dialyzed at $4^{\circ} \mathrm{C}$ for 2 h against buffer $\mathrm{C}(20 \mathrm{mM}$ Tris- $\mathrm{HCl}, \mathrm{pH} 8.0,40 \mathrm{mM}$ imidazole, and $300 \mathrm{mM} \mathrm{NaCl})$. The protein was passed through the Ni-NTA column equilibrated with buffer $\mathrm{C}$. The protein was loaded onto a HiTrap Heparin HP column (GE Healthcare) equilibrated with buffer D (20 mM Tris- $\mathrm{HCl}, \mathrm{pH} 8.0$, and $0.3 \mathrm{M} \mathrm{NaCl})$. The protein was eluted with a linear gradient of $0.3-2 \mathrm{M} \mathrm{NaCl}$. The purified proteins were stored at $-80^{\circ} \mathrm{C}$ until use. The mutations were introduced by a PCR-based method, and the sequences were confirmed by DNA sequencing. The sgRNA was transcribed in vitro with T7 RNA polymerase, using a partially doublestranded DNA template (Supplementary Tables 1 and 2). The transcribed RNA was purified by $8 \%$ denaturing (7 M urea) polyacrylamide gel electrophoresis.

\section{In vitro cleavage experiments}

The pUC119 plasmid, containing the 23-nt target sequence and the PAMs, was used as the substrate for in vitro cleavage experiments (Supplementary Table 1). The linearized pUC119 plasmid (100 ng, $4.7 \mathrm{nM})$ was incubated at $37^{\circ} \mathrm{C}$ for $0.5-5$ min with the CjCas9-sgRNA complex (50 nM) in $10 \mu \mathrm{L}$ of reaction buffer, containing $20 \mathrm{mM}$ HEPES, pH 7.5, $100 \mathrm{mM} \mathrm{KCl}, 2 \mathrm{mM} \mathrm{MgCl}_{2}, 1 \mathrm{mM}$ DTT, and 5\% glycerol. The reaction was stopped by the addition of quench buffer, containing EDTA ( $20 \mathrm{mM}$ final concentration) and Proteinase $\mathrm{K}(40 \mathrm{ng})$. The reaction products were resolved, visualized, and quantified with a MultiNA microchip electrophoresis device (SHIMADZU).

\section{PAM discovery assay}

The PAM discovery assays were performed, as previously described ${ }^{20}$. A library of pUC119 plasmids containing eight randomized nucleotides downstream of the target sequence was incubated at $37^{\circ} \mathrm{C}$ for 5 min with the CjCas9-sgRNA (22-nt guide) complex (50 nM), in $50 \mu \mathrm{L}$ of the reaction buffer. The reactions were quenched by the addition of Proteinase K, and then purified using a Wizard DNA Clean-Up System 
(Promega). The purified DNA samples were amplified for 25 cycles, using primers containing common adapter sequences (Supplementary Table 2). After column purification, each PCR product ( $5 \mathrm{ng}$ ) was subjected to a second round of PCR for 15 cycles, to add custom Illumina TruSeq adapters and sample indices. The sequencing libraries were quantified by qPCR (KAPA Biosystems), and then subjected to paired-end sequencing on a MiSeq sequencer (Illumina) with $20 \%$ PhiX spike-in (Illumina). The sequencing reads were demultiplexed by primer sequences and sample indices, using NCBI Blast+ (version 2.8.1) with the blastn-short option. For each sequencing sample, the number of reads for every possible 8-nt PAM sequence pattern $\left(4^{8}=65,536\right.$ patterns in total) was counted and normalized by the total number of reads in each sample. For a given PAM sequence, the enrichment score was calculated as $\log _{2}$-fold enrichment as compared to the untreated sample. PAM sequences with enrichment scores of 2.0 or less were used to generate the sequence logo representation, using WebLogo (version 3.7.1) ${ }^{29}$.

\section{Genome- and baseediting analyses in human cells}

Genome- and base-editing analyses were performed in triplicate, according to the protocol described previously ${ }^{30}$. Briefly, HEK293Ta cells were maintained in DMEM (Sigma) supplemented with $10 \%(\mathrm{v} / \mathrm{v})$ fetal bovine serum (FBS) (Thermo Fisher Scientific) and $1 \%$ Penicillin-Streptomycin (Sigma), at $37^{\circ} \mathrm{C}$ in a $0.05 \% \mathrm{CO}_{2}$ atmosphere. HEK239Ta cells were seeded at $5 \times 10^{3}$ cells per well in collagen I-coated 96 -well plates, $24 \mathrm{~h}$ prior to transfection. HEK239Ta cells were transfected with a CjCas9 plasmid or a CjCas9derived base-editor plasmid (120 ng) and an sgRNA plasmid (40 ng), using Polyethylenimine Max (Polysciences) $(1 \mathrm{mg} / \mathrm{mL}, 0.5 \mu \mathrm{L})$ in PBS $(50 \mu \mathrm{L})$. The cells were harvested 3 days after transfection, treated with $50 \mathrm{mM} \mathrm{NaOH}(100 \mu \mathrm{L})$, incubated at $95^{\circ} \mathrm{C}$ for $10 \mathrm{~min}$, and then neutralized with $1 \mathrm{M} \mathrm{Tris}-\mathrm{HCl}$, $\mathrm{pH} 8.0(10 \mu \mathrm{L})$. The obtained genomic DNA was subjected to two rounds of PCR, to prepare the library for high-throughput amplicon sequencing. Genomic regions targeted by sgRNAs were PCR-amplified to add custom primer-landing sequences (Supplementary Tables 2 and 3). The PCR products were purified by AMPure XP magnetic beads (Agencourt), and then subjected to a second round of PCR to attach the custom Illumina TruSeq adapters with sample indices. After size-selection by agarose gel electrophoresis and column purification, the sequencing libraries were quantified using a KAPA Library Quantification Kit Illumina (KAPA Biosystems), multiplexed, and subjected to paired-end sequencing (600 cycles), using a MiSeq sequencer (Illumina) with 20\% PhiX spike-in (Illumina). The sequencing reads were demultiplexed, based on sample indices and primer sequences. Using NCBI BLAST+ (version 2.6.0) with the blastn-short option, the sequencing reads were mapped to the reference sequences to identify indels and substitutions in the target regions. To remove common PCR errors and somatic mutations, sequencing reads with the same mutations observed in the control samples (null vector transfection) were removed, and then the mutation frequencies of the target sites were normalized by subtracting those of the same mutations observed in the control samples.

\section{Quantification and statistical analyses}

In vitro cleavage experiments were performed at least three times. Data are shown as mean \pm s.d. $(n=3)$. Kinetics data were fitted with a one-phase exponential association curve, using Prism (GraphPad). 


\section{Declarations}

Acknowledgements

This work was supported by JSPS KAKENHI Grant Numbers 18H02384 (H.N.) and 18K19284 (H.N.), and AMED Grant Number JP19am0401005 (N.Y., H.N., and O.N.).

\section{Author contributions}

R.N. performed biochemical experiments with assistance from S.O. and H.N.; S.I., H.M., M.T., H.A., and N.Y. performed cell biological experiments; R.N. and H.N. wrote the manuscript with assistance from S.I., H.M., N.Y., and O.N.; H.N. and O.N. supervised all of the research.

\section{Competing interests}

O.N. is a co-founder, board member, and scientific advisor for Modalis and Curreio.

\section{References}

1. Jinek, M. et al. A programmable dual-RNA-guided DNA endonuclease in adaptive bacterial immunity. Science 337, 816-821 (2012).

2. Hille, F. et al. The biology of CRISPR-Cas: backward and forward. Cell 172, 1239-1259 (2018).

3. Cong, L. et al. Multiplex genome engineering using CRISPR/Cas systems. Science $\mathbf{3 3 9}$, 819-823 (2013).

4. Sternberg, S.H., Redding, S., Jinek, M., Greene, E.C. \& Doudna, J.A. DNA interrogation by the CRISPR RNA-guided endonuclease Cas9. Nature 507, 62-67 (2014).

5. Doudna, J.A. The promise and challenge of therapeutic genome editing. Nature 578, 229-236 (2020).

6. Anzalone, A.V., Koblan, L.W. \& Liu, D.R. Genome editing with CRISPR-Cas nucleases, base editors, transposases and prime editors. Nat Biotechno/ 38, 824-844 (2020).

7. Fonfara, I. et al. Phylogeny of Cas 9 determines functional exchangeability of dual-RNA and Cas 9 among orthologous type II CRISPR-Cas systems. Nucleic Acids Res 42, 2577-2590 (2014).

8. Kim, E. et al. In vivo genome editing with a small Cas9 orthologue derived from Campylobacter jejuni. Nat Commun 8, 14500 (2017).

9. Koo, T. et al. Functional rescue of dystrophin deficiency in mice caused by frameshift mutations using Campylobacter jejuni Cas9. Mol Ther 26, 1529-1538 (2018).

10. Jo, D.H. et al. Long-term effects of in vivo genome editing in the mouse retina using Campylobacter jejuni Cas9 expressed via adeno-associated virus. Mol Ther 27, 130-136 (2019).

11. Lee, J.Y. et al. Efficient and specific generation of knockout mice using Campylobacter jejuni CRISPR/Cas9 system. Biochem Biophys Rep 22, 100752 (2020). 
12. Yamada, M. et al. Crystal structure of the minimal Cas 9 from Campylobacter jejuni reveals the molecular diversity in the CRISPR-Cas9 systems. Mol Cell 65, 1109-1121 e1103 (2017).

13. Anders, C., Niewoehner, O., Duerst, A. \& Jinek, M. Structural basis of PAM-dependent target DNA recognition by the Cas9 endonuclease. Nature 513, 569-573 (2014).

14. Komor, A.C., Kim, Y.B., Packer, M.S., Zuris, J.A. \& Liu, D.R. Programmable editing of a target base in genomic DNA without double-stranded DNA cleavage. Nature 533, 420-424 (2016).

15. Nishida, K. et al. Targeted nucleotide editing using hybrid prokaryotic and vertebrate adaptive immune systems. Science 353, aaf8729 (2016).

16. Gaudelli, N.M. et al. Programmable base editing of $A * T$ to $G * C$ in genomic DNA without DNA cleavage. Nature 551, 464-471 (2017).

17. Kim, Y.B. et al. Increasing the genome-targeting scope and precision of base editing with engineered Cas9-cytidine deaminase fusions. Nat Biotechno/35, 371-376 (2017).

18. Komor, A.C. et al. Improved base excision repair inhibition and bacteriophage Mu Gam protein yields C:G-to-T:A base editors with higher efficiency and product purity. Sci Adv 3, eaao4774 (2017).

19. Hirano, H. et al. Structure and engineering of Francisella novicida Cas9. Cell 164, 950-961 (2016).

20. Nishimasu, H. et al. Engineered CRISPR-Cas9 nuclease with expanded targeting space. Science $\mathbf{3 6 1}$, 1259-1262 (2018).

21. Ran, F.A. et al. In vivo genome editing using Staphylococcus aureus Cas9. Nature 520, 186-191 (2015).

22. Hirano, S. et al. Structural basis for the promiscuous PAM recognition by Corynebacterium diphtheriae Cas9. Nat Commun 10, 1968 (2019).

23. Karvelis, T. et al. Rapid characterization of CRISPR-Cas9 protospacer adjacent motif sequence elements. Genome Biol 16, 253 (2015).

24. Gao, L. et al. Engineered Cpf1 variants with altered PAM specificities. Nat Biotechno/35, 789-792 (2017).

25. Kleinstiver, B.P. et al. Engineered CRISPR-Cas12a variants with increased activities and improved targeting ranges for gene, epigenetic and base editing. Nat Biotechnol 37, 276-282 (2019).

26. Leenay, R.T. et al. Identifying and visualizing functional PAM diversity across CRISPR-Cas systems. Mol Cell 62, 137-147 (2016).

27. Nishimasu, H. et al. Crystal structure of Cas9 in complex with guide RNA and target DNA. Cell 156, 935-949 (2014).

28. Li, X. et al. Programmable base editing of mutated TERT promoter inhibits brain tumour growth. Nat Cell Biol 22, 282-288 (2020).

29. Crooks, G.E. et al. WebLogo: A sequence logo generator. Genome Res 14, 1188-1190 (2004).

30. Sakata, R.C. et al. Base editors for simultaneous introduction of C-to-T and A-to-G mutations. Nat Biotechnol 38, 865-869 (2020). 
a

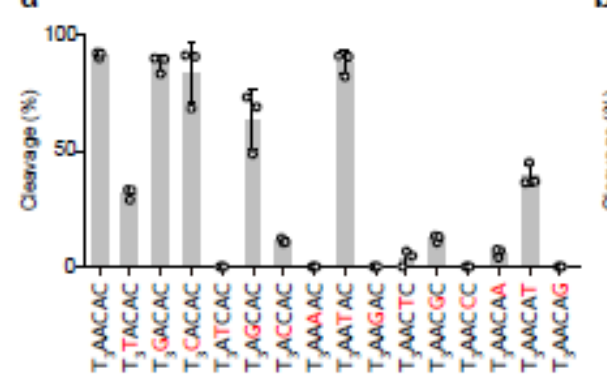

c

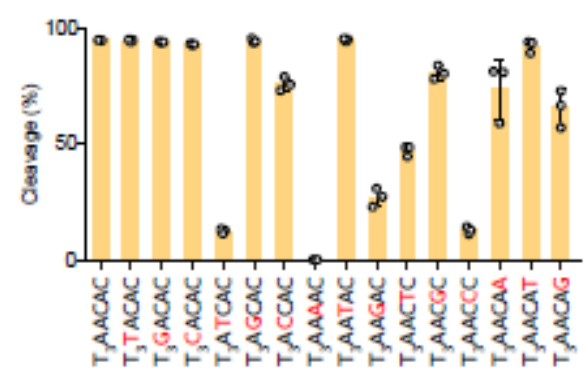

b

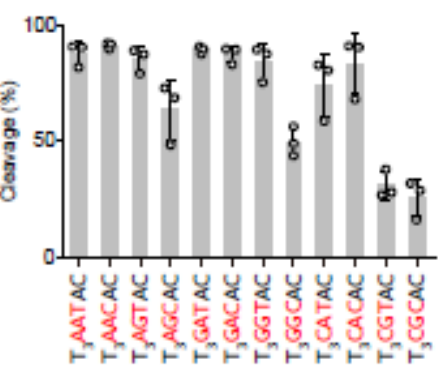

d

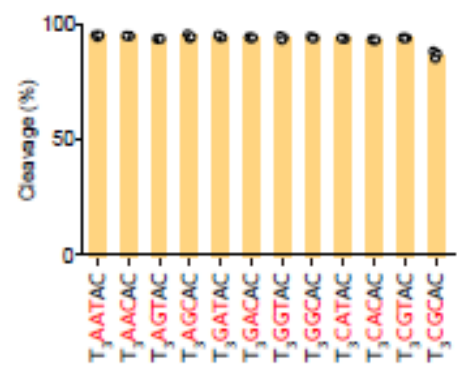

e

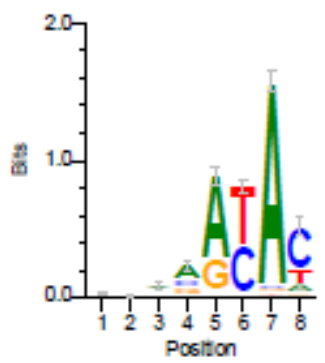

$f$

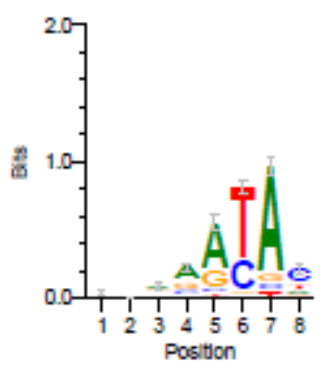

g

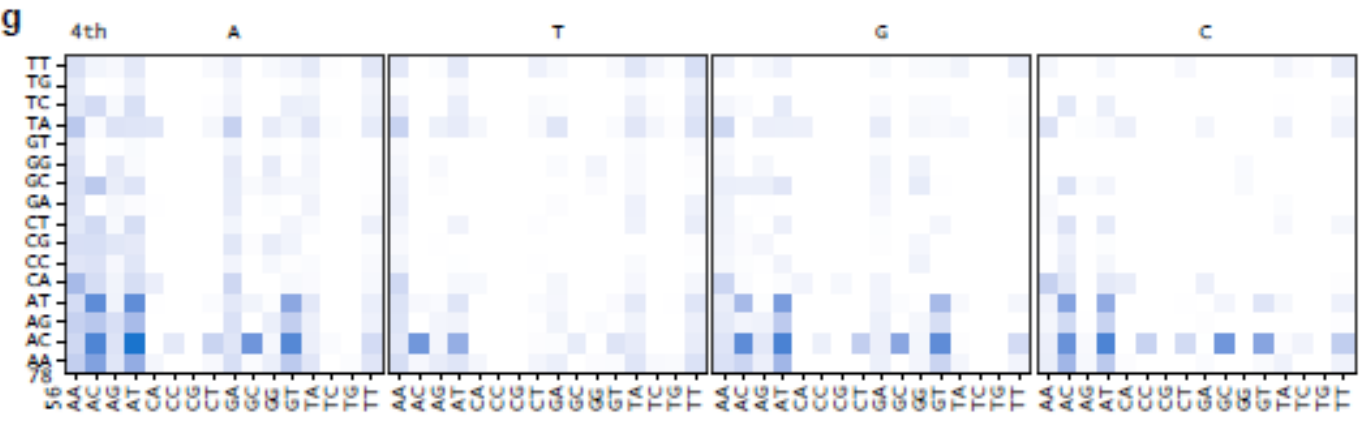

$\mathrm{h}$

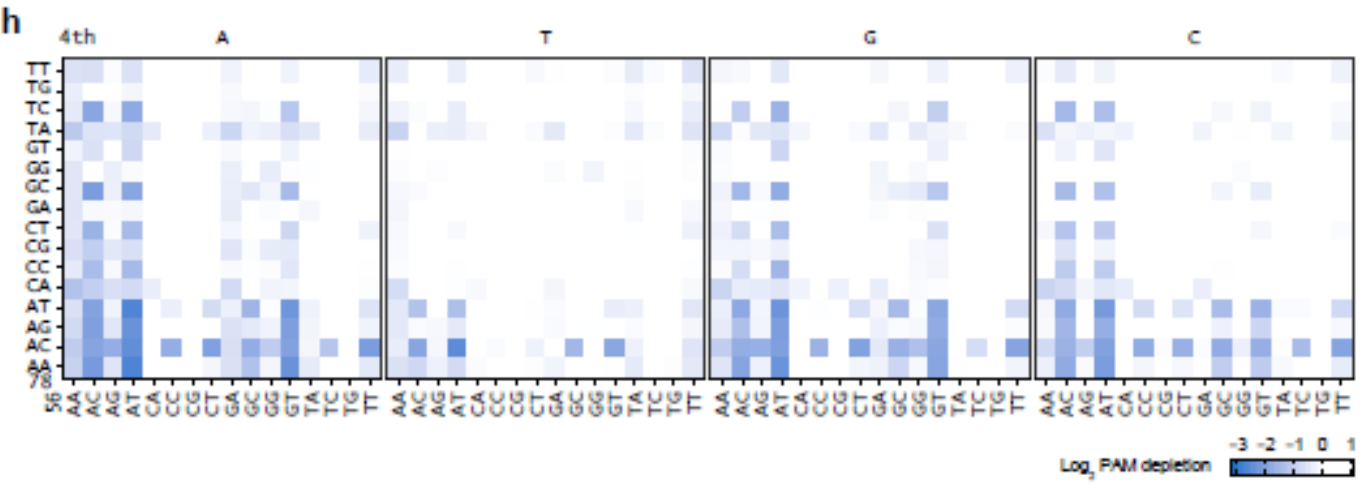

Figure 1

In vitro cleavage activities of CjCas9 and enCjCas9. (a-d) In vitro cleavage activities of CjCas9 (a and b) and enCjCas9 (c and d) toward DNA targets with different PAMs. The linearized plasmid targets were incubated with the CjCas9-sgRNA complex at $37^{\circ} \mathrm{C}$ for 2 min. Data are mean \pm s.d. $(n=3) .(e, f)$ Sequence logos of CjCas9 (e) and enCjCas9 (f) obtained from the in vitro PAM discovery assay. $(g, h) 2 D$ PAM profiles of CjCas9 $(\mathrm{g}$ ) and enCjCas9 $(\mathrm{h})$ obtained from the in vitro PAM discovery assay. 
a

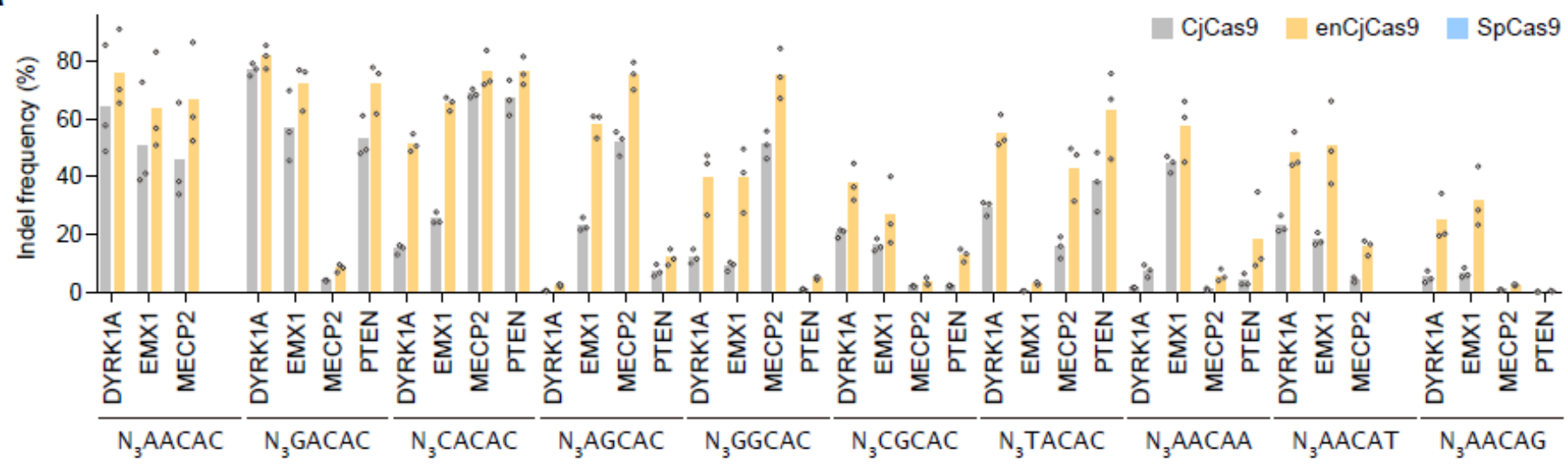

b

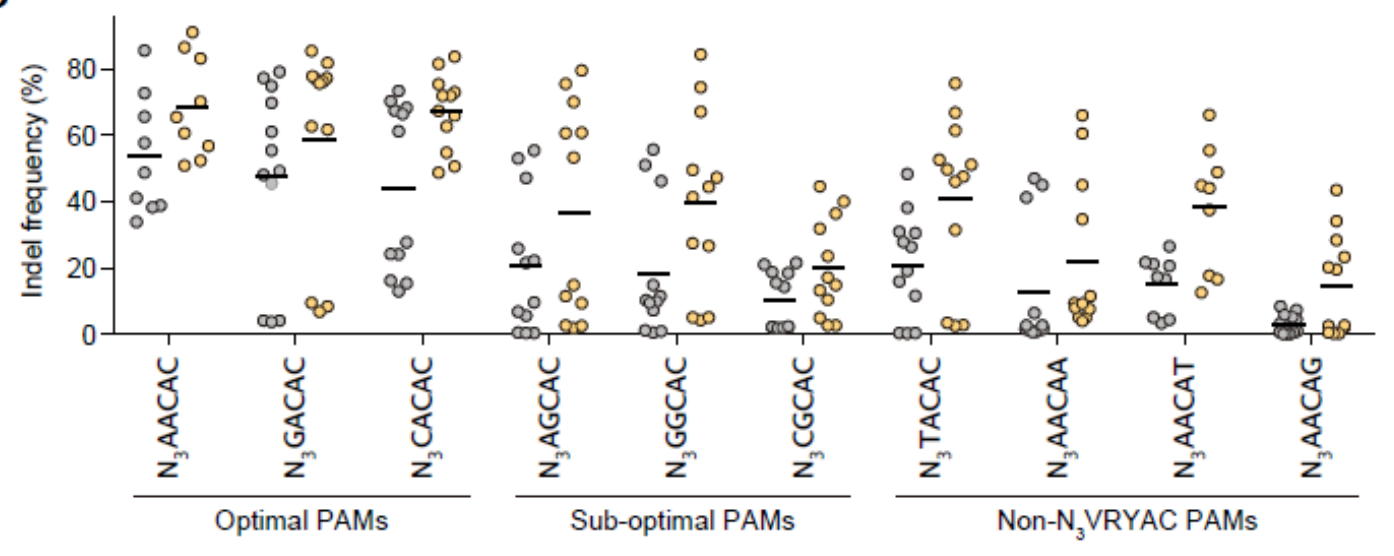

C

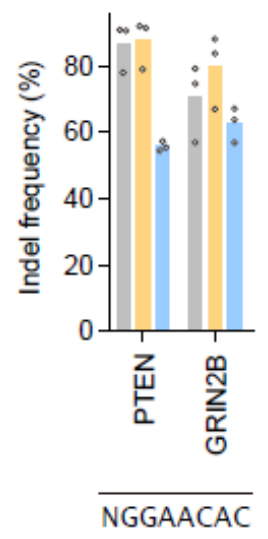

Figure 2

Genome editing by CjCas9 and enCjCas9. (a) Indel formation by CjCas9 (gray) and enCjCas9 (orange) at endogenous target sites in HEK293Ta cells $(n=3)$. (b) Summary of the genome-editing efficiencies of CjCas9 (gray) and enCjCas9 (orange) in (a). Bars indicate medians. (c) Indel formation by CjCas9 (gray), enCjCas9 (orange), and SpCas9 (blue) at endogenous target sites in HEK293Ta cells $(n=3)$. 

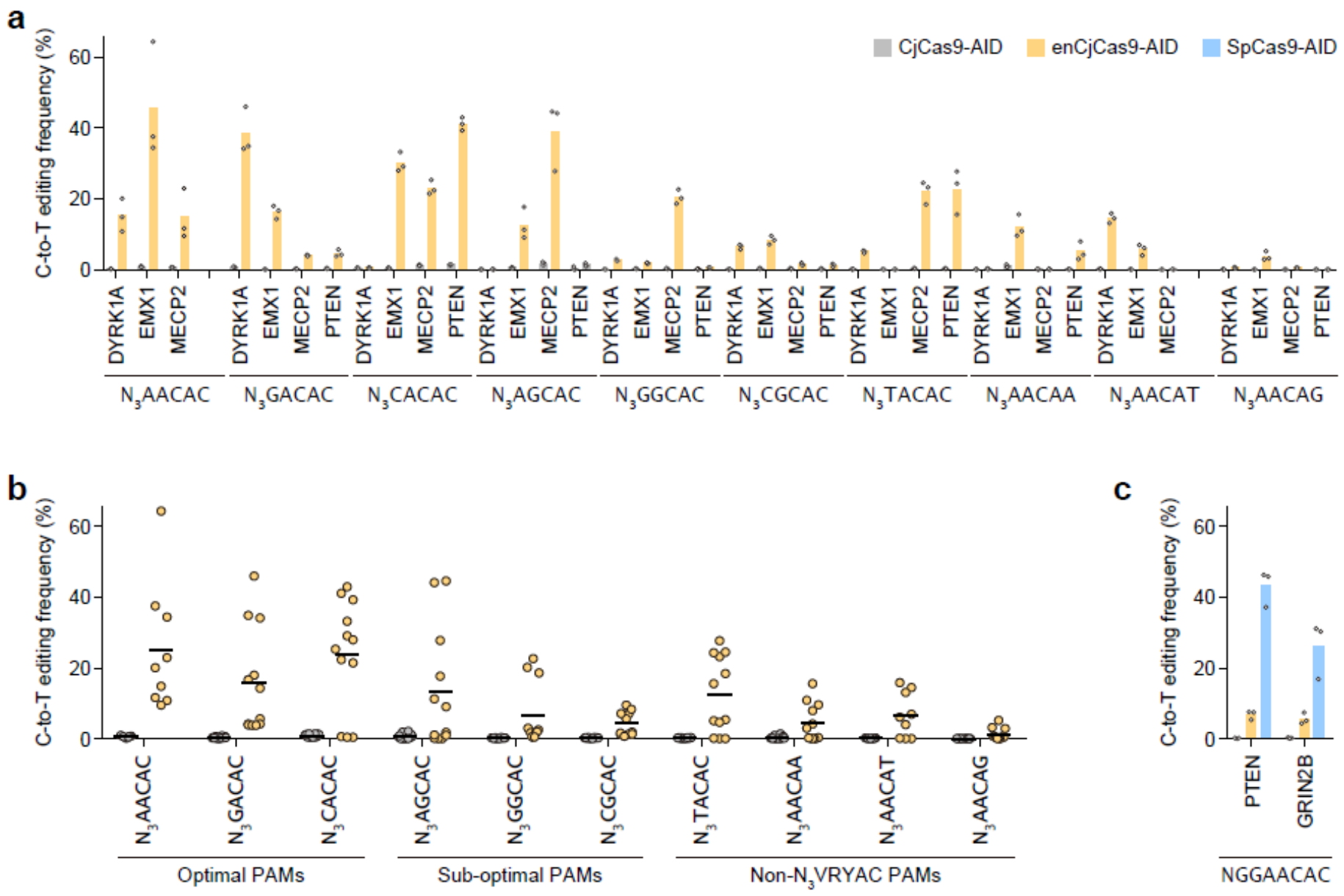

\section{Figure 3}

Base editing by CjCas9-AID and enCjCas9-AID. (a) C-to-T conversion by CjCas9-AID (gray) and enCjCas9AID (orange) at endogenous target sites in HEK293Ta cells $(n=3)$. (b) Summary of the base-editing efficiencies of CjCas9-AID (gray) and enCjCas9-AID (orange) in (a). Bars indicate medians. (c) C-to-T conversion by CjCas9-AID (gray), enCjCas9-AID (orange), and SpCas9-AID (blue) at endogenous target sites in HEK293Ta cells $(n=3)$.

\section{Supplementary Files}

This is a list of supplementary files associated with this preprint. Click to download.

- CjCas9210510sup.pdf

- SupplementaryFiguresLegendsandTables.docx 